\title{
On Analytical Comparative Study Considering Quantified Learning Creativity Analogy versus Ant Colony Intelligence
}

\author{
Hassan Mustafa ${ }^{1}$ and Fadhel Ben Tourkia ${ }^{2}$
}

\begin{abstract}
This piece of research introduces an investigational systematic study of an interdisciplinary challenging phenomenon observed in natural world. Interestingly, this study belongs to the two emerging fields of nature-inspired computing (NIC) and computational intelligence (CI) with focusing on the physics- and biology-based approaches and algorithms .Herein, by more details this article concerned with the conceptual analysis and evaluation of quantified learning creativity phenomenon via simulation and modeling of two diverse natural biological systems (human \& nonhuman creatures). More precisely, it is associated to diverse aspects of measurable behavioral learning performance of both biological systems. Therefore, this paper introduces comparative analogy between two diverse biological behavioral systems considering quantification of learning creativity. Referring to, the definition of Swarm intelligence which considered as a relatively new discipline that deals with the study of self-organizing processes both in nature and in artificial systems. Researchers in ethology and animal behavior have proposed many models to explain interesting aspects of social insect behavior such as self-organization and shapeformation. Accordingly, the presented study observed during human interactive tutoring/learning processes with natural environment. Versus ecological behavioral learning of swarm intelligence agents (Ants), while performing foraging process . Systematic investigational study of quantified human learning creativity phenomenon is an interdisciplinary, challenging, and interesting educational issue. At education field practice (classrooms), while face to face tutoring sessions are performed, learning creativity phenomenon is detectable via bidirectional feedback between teacher and pupil. In short, this research work adopts comparative study of simulation and modeling for educational creativity issue considering two disciplinary approaches are namely: swarm intelligence, and neural networks. Both simulated realistically for systematic investigational modeling of creatures' creativity phenomenon observed in nature. Presented creativity models mainly consider observed behavioral learning of ant colony system in addition to in field educational classrooms. Conclusively, presented results herein, for both swarm intelligence and neural networks models seemed to be well promising for future more elaborate, systematic, and innovative research in evaluation of human learning creativity phenomenon regarding (NIC) and (CI).
\end{abstract}

Keywords - Artificial Neural Networks Modeling, Ant Colony Optimization, Noisy Learning Enviornment, Optical Character Recognition, Swarm Intelligence.

Hassan Mustafa ${ }^{1}$ Computer Engineering Department, Al-Baha Private College of Sciences Al-Baha, Kingdom of Saudi Arabia.On leave from Banha University (EGYPT)

Fadhel Ben Tourkia ${ }^{2}$, Computer Engineering Department, Al-Baha Private College of Sciences Al-Baha, Kingdom of Saudi Arabia

\section{INTRODUCTION}

THIS research work deals with an interdisciplinary, interesting, and challenging problem associated with two emerging research fields namely: nature inspired computing (NIC) and computational intelligence (CI), [1]. Furthermore, by referring to [2], it is announced therein: Natural Computing is that field of research concerned with investigation of human-designed computing inspired by nature as well as computing taking place in nature by social insects. That is, it investigates models and computational techniques inspired by nature, and also it investigates, in terms of information processing, phenomena taking place in nature. Referring to the instructional methodology of face to face tuition, bidirectional interactive phenomenon observed between teacher and pupil applied in classrooms. Herein, adopted realistic simulation of that tuition methodology is performed by using $\mathrm{ANN}^{\mathrm{s}}$ supervised learning modeling (Error correction learning rule). Interestingly, by referring to what has been announced by at [3], in the context of attempting to demystify the term Swarm Intelligence (SI). Prof Franks said that although apes could imitate each other, it was a one-way process. According to the accepted definition of teaching in animal behavior, an individual is a teacher if it modifies its behavior in the presence of a naïve observer, at some initial cost to itself, in order that the pupil can learn more quickly. Prof Franks said: "We also believe that true teaching always involves feedback in both directions between the teacher and the pupil. In other words, the teacher provides information or guidance for the pupil at a rate suited to the pupil's abilities, and the pupil signals to the teacher when parts of the 'lesson' have been assimilated". Moreover, Tandem running in the Temnothorax ants met these criteria, he said. Ants laid trails of chemicals, called pheromones, to guide other colony members to food but only they could read the signals. Consequently, the ANN $\underline{s}$ supervised learning model is well relevant for realistic simulation of adopt an intelligent teaching technique known as tandem running. That approach has been performed by considering ant colony system of Temnothorax albipennis (formerly Leptothorax albipennis).

By other wording, in brief, this paper adopts the analytical and comparative study for the analogy between two distinctly diverse behavioral biological systems. They both characterized by quantified learning creativity versus behavioral swarm intelligence, respectively. That associated to humans (face to face tutoring pupils in classrooms) versus intelligence of social insects (ACS solving TSP 
optimally),[4][5][6][7]. Furthermore, the paper presents observed effect of noisy environmental nature on performance of learning creativity as well as the behavioral ACS intelligence, [8][9][10][11]. Additionally, the issue of quantified learning creativity has been studied using realistic ANN modeling at some published papers $[12][13][14][15][16]$. The rest of this paper after the previous introductory first section, is organized in five sections as follows. At the next second section, a revision of conceptual aspects' for instructional process considering modeling of learning paradigms modeling using ANN is introduced. At the third section, the effect of environmental noise on learning performance is presented. Revision for some algorithms to solve TSP using ACS is introduced at the fourth section. Some of obtained interesting simulation results are given at the fifth section. Finally, at the sixth, some of conclusive remarks and suggestions for future work are introduced.

\section{LEARNING MODELS' REVISION}

This section introduces a revision for the conceptual basis of realistic interactive modeling of teaching/learning process via the three subsections denoted as follows : $(A, B$, and $C)$. At the subsection $A$, relation between an artificial and biological single neuron model is considered along with illustration using basic mathematical formulae. A generalized brief overview of the block diagram describing interactive teaching/learning process that considers (face to face tuition) is given. at subsection $B$. At subsection $C$, the detailed mathematical formulation introduced for both bidirectional communication between any teacher and his learners. That two learning paradigms are namely: guided with a teacher (supervised) and without a teacher equivalently to self-organized (unsupervised). [17][18].

\section{A. Simplified Modeling for a Single Biological Neurone}

Realistic modeling of biological neural system, is considered via distributed parallel information processing. However, a single biological neuron is the basic building block of any neural system. In more details, by referring to Fig., inside any single neuron performed information processing transferred among three basic structural components (Dendrites, Soma, and Axon) [19].

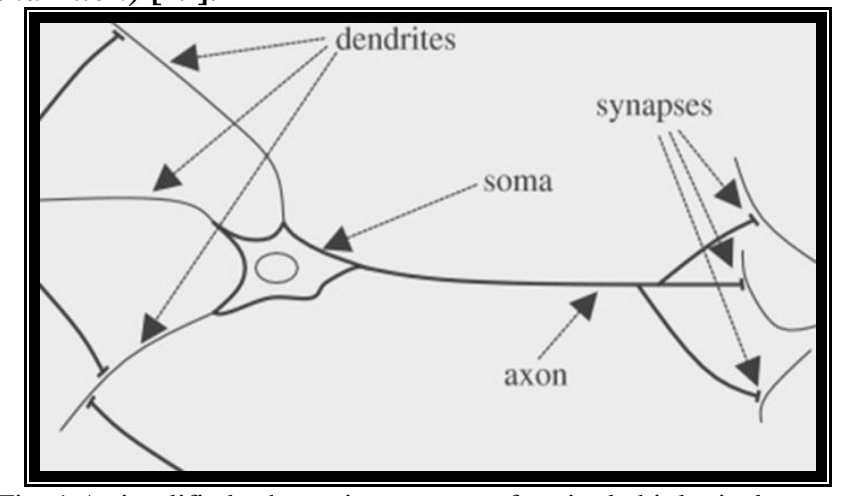

Fig. 1 A simplified schematic structure of a single biological neuron adapted from [19]

Accordingly, realistic neuron model composed of three basic elements of that model given (by referring to the graphical presentation shown at Fig.2. in below) as follows:
- A set of weights, each of which is characterized by a strength of its own. A signal $\mathrm{x}_{\mathrm{i}}$ connected to neuron $\mathrm{k}$ is multiplied by the weight $\mathrm{w}_{\mathrm{ki}}$. The weight of an artificial neuron may lie in a range that includes negative as well as positive values.

- An adder for summing the input signals, weighted by the respective weights of the neuron.

- An activation function for limiting the amplitude of the output of a neuron. It is also referred to as transfer function which squashes the amplitude range of the output signal to some finite value. Two types of the sigmoid transfer (activation)functions are commonly used in ANN applications. First one is the logistic sigmoid and the second other function is the odd sigmoid. There values are given at any arbitrary time instant (n) by equations (3) \& (4) respectively.

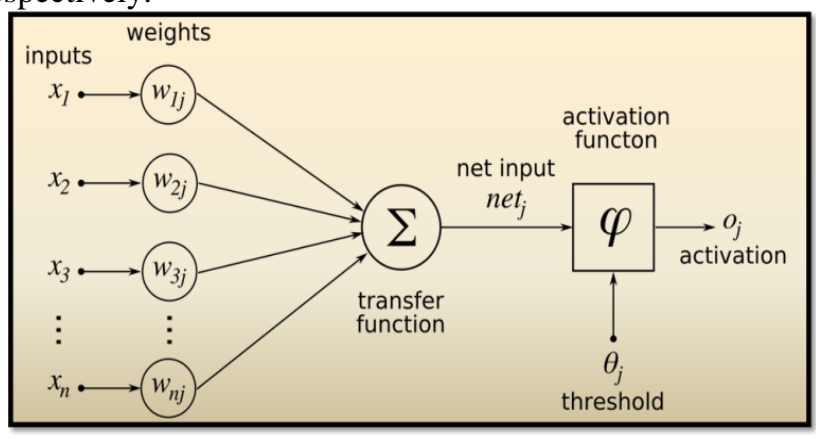

Fig. 2. A single neuron (k) model coupled with synaptic weights from other neurons $w_{k i}(\mathrm{i}, \ldots \ldots, \mathrm{p})$.

$\mathrm{V}_{\mathrm{k}}=\sum_{i=1}^{p} w_{k i} x_{i}$

$\mathrm{y}_{\mathrm{k}}=\varphi\left(\mathrm{V}_{\mathrm{k}}+\theta_{\mathrm{k}}\right)$

The odd sigmoid function seemed to be well relevant for realistic simulation of learning brain performance as if this function input stimulus equals zero results in obtaining no output (zero).

$\mathrm{y}_{\mathrm{k}}(\mathrm{n})=\varphi\left(\mathrm{V}_{\mathrm{k}}(\mathrm{n})\right)=1 /\left(1+\mathrm{e}_{\mathrm{k}}^{-\lambda \mathrm{v}(\mathrm{n})}\right)$

$\mathrm{y}_{\mathrm{k}}(\mathrm{n})=\varphi\left(\mathrm{V}_{\mathrm{k}}(\mathrm{n})\right)=\left(1-\mathrm{e}_{\mathrm{k}}^{-\lambda \mathrm{v}}(\mathrm{n}) /\left(1+\mathrm{e}_{\mathrm{k}}^{-\lambda \mathrm{v}}(\mathrm{n})\right.\right.$

\section{B. Modeling Of Interactive Learning Processes}

Face to face tuition is illustrated as an interactive learning processes presented at Fig. 1. Inputs to the neural network teaching model are provided as a signal provided by environmental stimuli (unsupervised learning). However, correction signal(s) in the case of learning using a teacher's guidance is/are given by output response(s) of the model that evaluated by either the environmental conditions (unsupervised learning) or by supervision of a teacher. Additionally, any tutor plays a role of improvement input data (stimulating learning pattern) by reducing the noise and redundancy of model pattern input. That is motivated by the tutor's experience while performing conventional (classical) learning. Consequently, the tutor provides the learning model with cleared data via maximizing of the signal to noise ratio [20]. Conversely, in the case of unsupervised/self-organized 
learning, which is based upon Hebbian learning rule [21], it is mathematically formulated by equation (11) presented at the next subsection $(C)$.

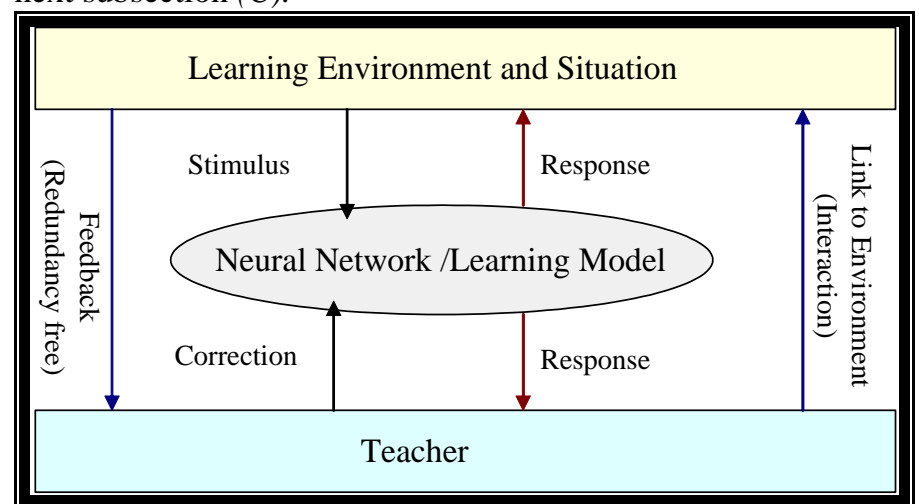

Fig. 3. Simplified view for interactive learning process $\{$ Adapted from $[22]\}$.

C. Mathematical Formulation Of Interactive Learning

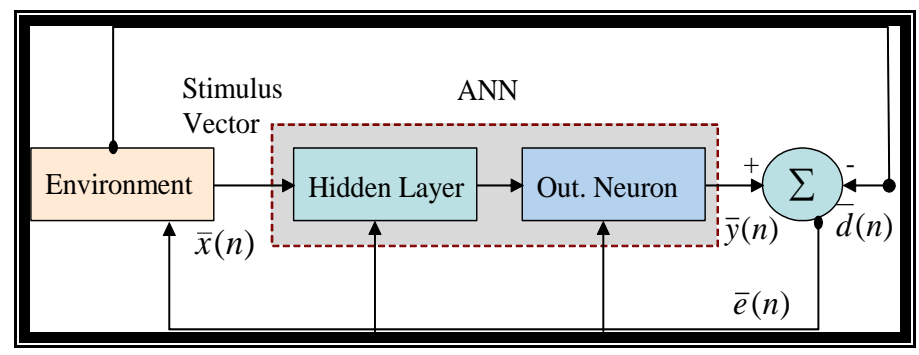

Fig. 4. Generalized ANN block diagram simulating two diverse learning paradigms adapted from [18].

At Figure 4., an interactive learning model through stimulating signals is well qualified in performing realistic simulation for evaluating learner's performance. This Figure illustrates inputs to the neural network learning model which provided by stimuli unsupervised learning environment[18]. The correction signal for the case of learning with a teacher is given by responses outputs of the model will be evaluated by either the environmental conditions (unsupervised learning) [23] or by the instructor. The instructor plays a role in improving the input data (stimulating learning pattern), by reducing noise and redundancy of learning model pattern input .In accordance with instructor's experience, he provides illustrated model with clear data by maximizing learning environmental signal to noise ratio [24].

By more details, referring to above Figure 4; the error vector $\bar{e}(n)$ at any time instant (n) observed during learning processes is given by:

$$
\bar{e}(n)=\bar{y}(n)-\bar{d}(n)
$$

Where $\bar{e}(n)=$ is the error correcting signal vector that is controlling adaptively the learning process outcome, $\bar{y}(n) \ldots \ldots$ is the obtained outcome (output) signal developed by ANN model, and $\bar{d}(n) \ldots \ldots$ is the desired vector or numerical value(s). Moreover, the following four equations are deduced:

$$
V_{\mathrm{k}}(n)=X_{j}(n) W_{k j}^{T}(n)
$$

$$
\begin{aligned}
& Y_{\mathrm{k}}(n)=\varphi\left(V_{\mathrm{k}}(n)\right)=\left(1-\mathrm{e}^{-\lambda V_{\mathrm{k}}(n)}\right) /\left(1+\mathrm{e}^{-\lambda V_{\mathrm{k}}(n)}\right) \\
& e_{\mathrm{k}}(n)=\left|d_{\mathrm{k}}(n)-y_{\mathrm{k}}(n)\right| \\
& W_{\mathrm{kj}}(n+1)=W_{\mathrm{kj}}(n)+\Delta W_{\mathrm{kj}}(n)
\end{aligned}
$$

Where $X$ is input vector and $W$ is the weight vector. $\varphi$ is the activation function. $Y$ is the output. $e_{\mathrm{k}}$ is the error value and $d_{\mathrm{k}}$ is the desired output. Note that $\Delta W_{\mathrm{kj}}(\mathrm{n})$ is the dynamical change of weight vector value. Above four equations are commonly applied for both learning paradigms: supervised (interactive learning with a tutor), and unsupervised (learning though student's self-study). The dynamical changes of weight vector value specifically for supervised phase is given by:

$$
\Delta W_{\mathrm{kj}}(n)=\eta e_{\mathrm{k}}(n) X_{j}(n)
$$

Where $\eta$ is the learning rate value during the learning process for both learning paradigms. At this case of supervised learning, instructor shapes child's behavior by positive/ negative reinforcement Also, Teacher presents the information and then students demonstrate that they understand the material. At the end of this learning paradigm, assessment of students' achievement is obtained primarily through testing results. However, for unsupervised paradigm, dynamical change of weight vector value is given by:

$\Delta W_{\mathrm{kj}}(n)=\eta Y_{\mathrm{k}}(n) X_{j}(n)$

Noting that $e_{\mathrm{k}}(\mathrm{n})$ equation (10) is substituted by $y_{\mathrm{k}}(\mathrm{n})$ at any arbitrary time instant (n) during the interactive learning process. Referring to Fig.1, the correction signal which provided by a tutor should take into consideration the noisy environmental level inside classrooms (such as noisy crowdedness appears . . In other words, that level is quantitatively measured as signal to noise $(\mathrm{S} / \mathrm{N})$ ratio or equivalently the additive noise power $(\sigma)$ to the ideally sensory clear signal. Consequently, the response time response measured by number of training cycles $(n)\{$ as defined at the subsection in the above $(B)$ by the two equations (10) \& (11)\}. Noting value of (n) should have been increased until reaching learning convergence instant, when:

$\Delta W_{\mathrm{kj}}(n)=0$

That above condition given by equation (8), could be fulfilled only if the desired output learning has been obtained after some number of training cycles (response time) in fulfillment of the two equations (10) \& (11).

\section{REVIEW OF NOISY ENVIRONMENTAL CONCEPTS [22]}

This section presents a comprehensive simulation findings reviewed form the observed concepts of environmental noise levels in nature [11]. It is worth noting that statistical variations(on the average) relating learning rate values versus corresponding learning convergence (response) time. That time is measured by the number of iteration cycles. Referring to Figure 5,obtained output results (of response time) corresponding to the learning rate values $(0.1,0.2,0.4,0.6$, and 0.8), are given respectively, as (330, 170, 120, 80, and 40) iteration training cycles (At TABLE I.) . Conclusively, convergence time (number of training cycles) is inversely proportional to the corresponding learning rate values. 
Referring to, Figure 6., an illustration by statistical distribution performance (Similarly, to the bell shape behavioral performance) is presented for the normalized obtained outcomes', considering various values of learning rate: $\eta(0.05,0.3$, and 0.5$)$

TABLE I

THE EFFECT OF LEARNING RATE VALUES ON CONVERGENCE LEARNING (RESPONSE)TIME\{ADAPTED FROM [22]\}

\begin{tabular}{||c||c||c||c||c||c||}
\hline $\begin{array}{c}\text { Learning } \\
\text { Rate Values ( } \square \square\end{array}$ & $\mathbf{0 . 1}$ & $\mathbf{0 . 2}$ & $\mathbf{0 . 4}$ & $\mathbf{0 . 6}$ & $\mathbf{0 . 8}$ \\
\hline \hline $\begin{array}{c}\text { Learning } \\
\text { Response Time } \\
\text { (\# cycles) }\end{array}$ & $\mathbf{3 3 0}$ & $\mathbf{1 7 0}$ & $\mathbf{1 2 0}$ & $\mathbf{8 0}$ & $\mathbf{4 0}$ \\
\hline
\end{tabular}

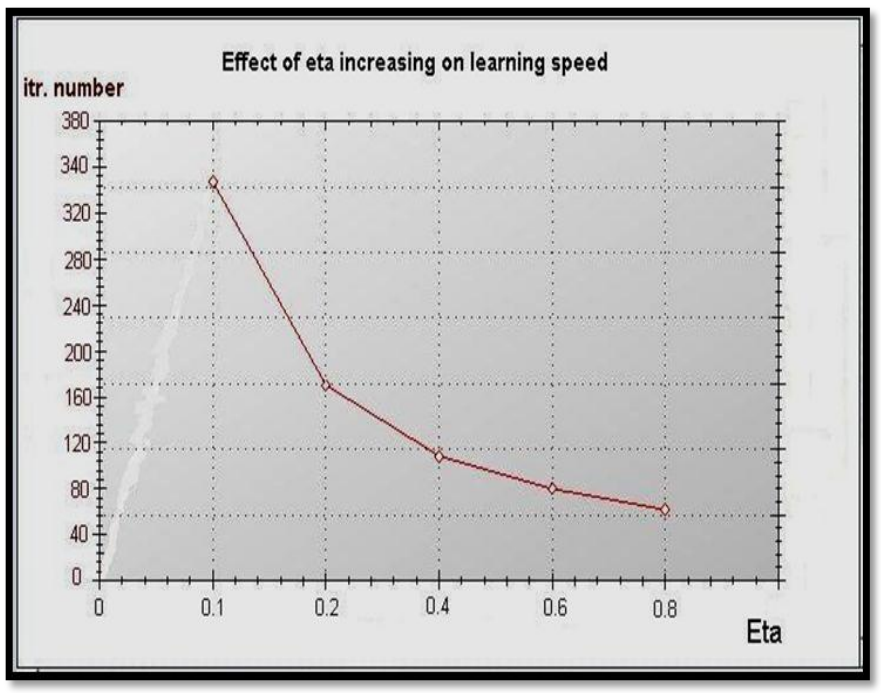

Fig. 5 Illustrates the average (of statistical distribution) for learning response time (number of iteration cycles) for different learning rate values $\eta$ (eta)

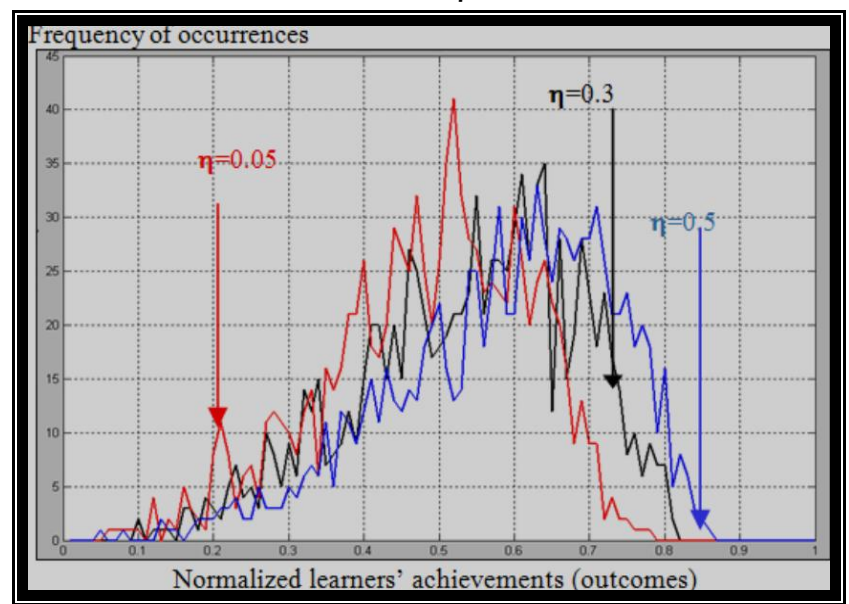

Fig. 6. Illustration of obtained normalized outcomes corresponding to three different learning rate values $\eta(0.05,0.3$, and

$$
\text { 0.5). }\{\text { Adapted from[22]\} }
$$

\section{B. Effect Of Noise On OCR Processes [24]}

In nature, optical character recognition OCR as well as pattern recognition processes observed to be carried out under no ideal environmental condition (under effect of noisy data). Interestingly, obtained simulation results for OCR
Under different environmental noisy levels are given in a tabulated form at TABLE II. Noting that, noise effect is measured by signal to noise ratio value $(\mathrm{S} / \mathrm{N})$ versus the number of training cycles $(\mathrm{T})$ till reaching learning convergence. Conclusively, relation between number of training cycles' values and noisy levels of environmental data (for the case of unsupervised learning paradigm) is illustrated well at Figure 7. Referring to that figure, learning convergence time $\mathrm{T}$ in cycles ( $\mathrm{n}$ ), inversely proportional to signal to noise ratio values, $(\mathrm{S} / \mathrm{N})$. learning convergence time has been presented at TABLE II. That table derived from, Figure 7. , at [22]. Conclusively, it is observed during interactive learning process that: teaching/learning environment with increasing $(\mathrm{S} / \mathrm{N})$ ratio results in decreasing of learning rate parameter value $\eta$.That explicitly computed as noise power value ( $\sigma$ ) [24].

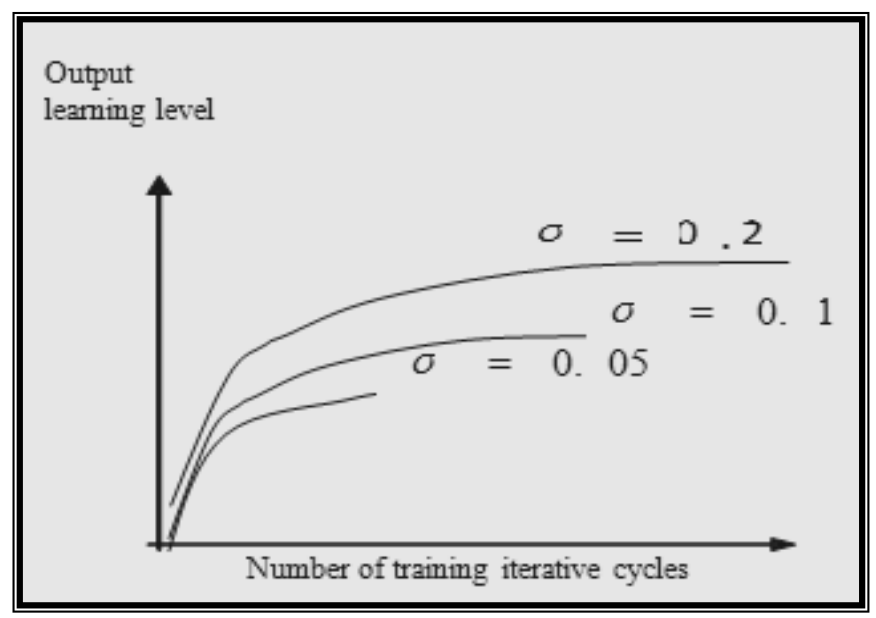

Fig.7. Relation between noise power ( $\sigma$ ) that represents non-properly prepared (noisy) learning process convergence, \{Adapted from [24] \}. TABLE II

THE EFFECT OF NOISY ENVIRONMENTAL LEARNING ON CONVERGENCE TIME, \{ADAPTED FROM [24]\}

\begin{tabular}{|c|c|c|c|}
\hline $\begin{array}{l}\text { Signal to Noise }(\mathrm{S} / \mathrm{N}) \text { Power Ratio of } \\
\text { Input Data }\end{array}$ & 5 & 10 & 20 \\
\hline $\begin{array}{l}\text { Noise Power in Learning } \\
\text { Environment } \square \square\end{array}$ & 0.2 & 0.1 & 0.05 \\
\hline $\begin{array}{l}\text { Convergence Learning Time (\# } \\
\text { cycles) }\end{array}$ & 82 & 62 & 47 \\
\hline
\end{tabular}




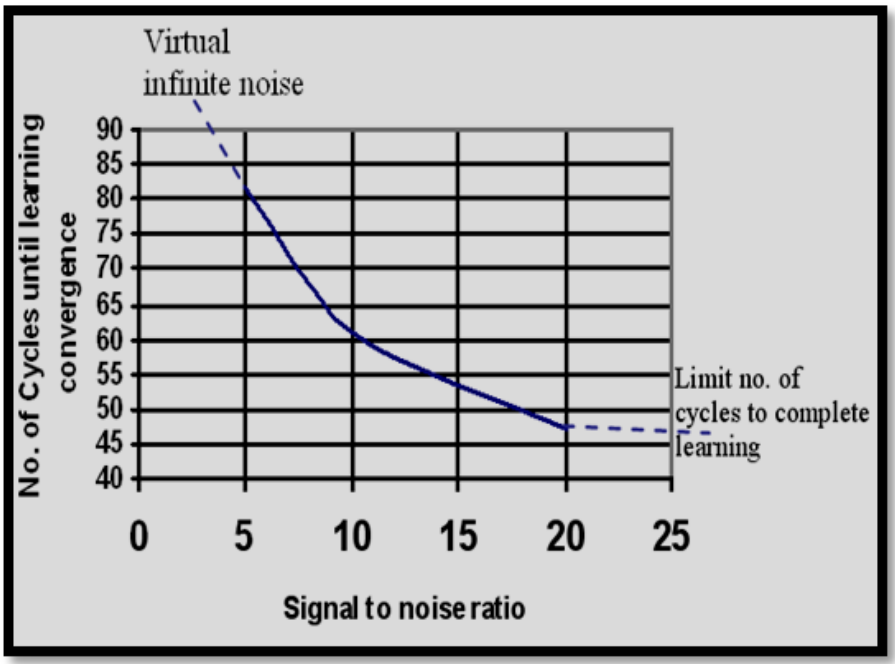

Fig. 8. Graphical presentation for learning performance under noisy conditions with reference to Table 1I. \{ adapted from [24]\}.

TABLE III

RELATION BETWEEN AVERAGE NO OF CYCLES NEEDED TO REACH OPTIMUM SOLUTION VERSUS SIGNAL TO NOISE RATIO VALUES [25].

\begin{tabular}{|l|l|l||l||}
\hline Signal to Noise Power Ratio of Input Data & 1 & 10 & 20 \\
\hline \hline Convergence to Opt. Sol. Time (\# cycles) & 100 & 50 & 30 \\
\hline
\end{tabular}

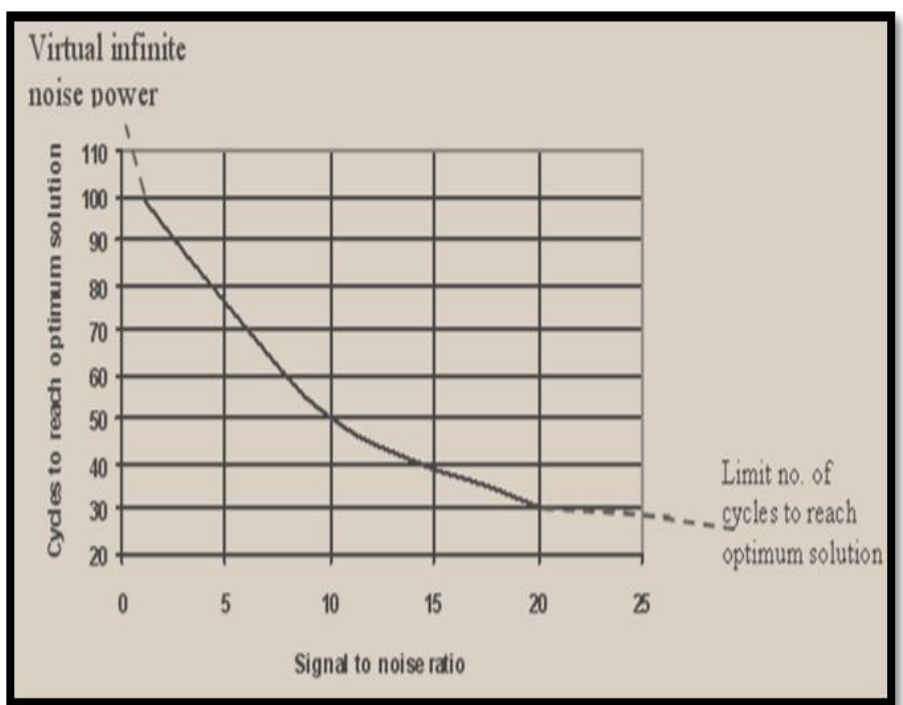

Fig. 9. Graphical presentation for reach optimal solution of TSP under noisy conditions [25]

\section{SOLVING OF TSP UsING ACS ALgORITHMS}

\section{A. Selection of Minimum Pathway Between Source and}

\section{Nest [26]}

Referring to Figure 10, in the case of bifurcation occurrence due to an existence of an obstacle at some point placed on the pathway between the nest site and that of the source, the transportation process of food (from food source) to food store (nest). is illustrated behavioral ants' responses shown at the simplified sketched figure considering the pheromone trail between nest and food source.

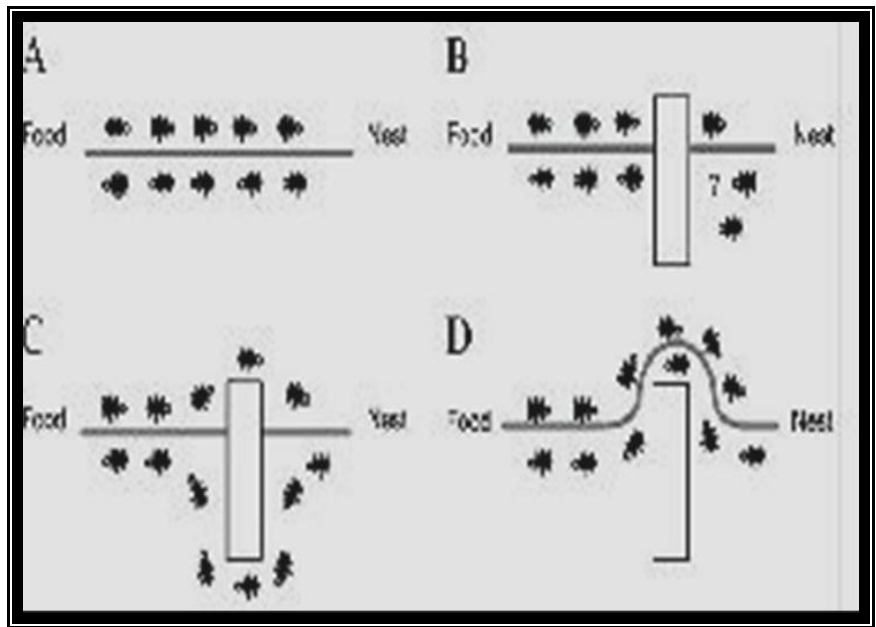

Fig. 10. Ant Behavior A. Ants in a pheromone trail between nest and food; B. An obstacle interrupts the trail; C. Ants find two paths to go around the obstacle; D. A new pheromone trail is formed along the shorter path $\{$ Adapted from[26]\}.

Interestingly, another type of ACS that is capable to converge to (accomplish) optimal solution of Traveling Salesman Problem (TSP) by adopting an autocatalytic ACS algorithm. That's with dependence upon different intercommunication levels among ACS agents (ants). The reached optimal solutions of TSP obtained by various speeds in accordance with inter-communication levels among ACS agents [27][28][29][30].

\section{B. Analogy between Supervised ANN Model Versus Tandam Learning}

Referring to [29], the two Figures (11\& 12), present two distinct models for either supervised learning ANN, or the ACS adopting tandem learning strategy, respectively. More precise words, both models has been provided by interactive leaning responses either by guided relationship for teacher/pupil tuition at (Figure 11), or by the environmental learning of leader/follower ants (Tandem running), at (Figure 12). Therefore, in case if the a teacher provides an interactive response, information or guidance correction is the stimulating signal for the pupil. Similarly, for ACS the interactive bidirectional response observed between leader and follower. Both cases are noticed to be formulated as (supervised learning) or equivalently (learning by a teacher) .That is given by the derived formula according to equation (10) at the above section II. 


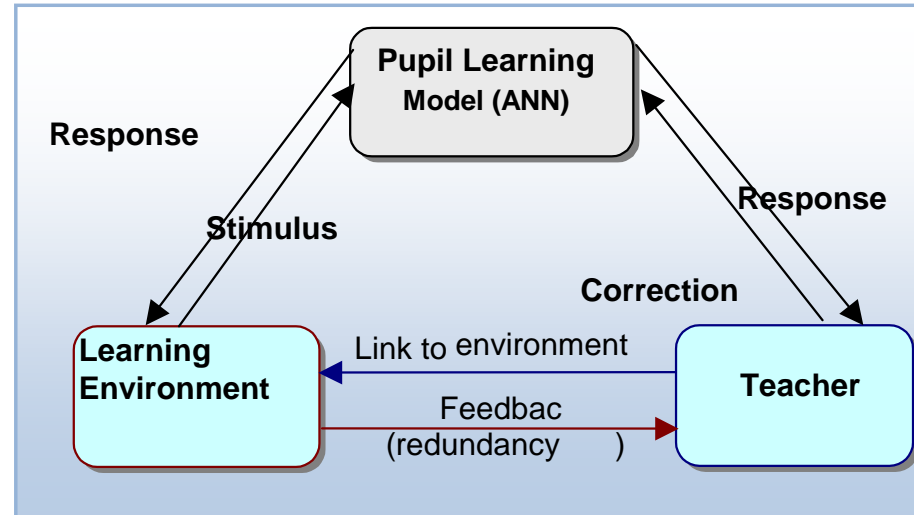

Fig. 11. A Schematic Diagram for (Supervised Learning Interaction Between a Teacher and his Pupil. ANN Based Model)

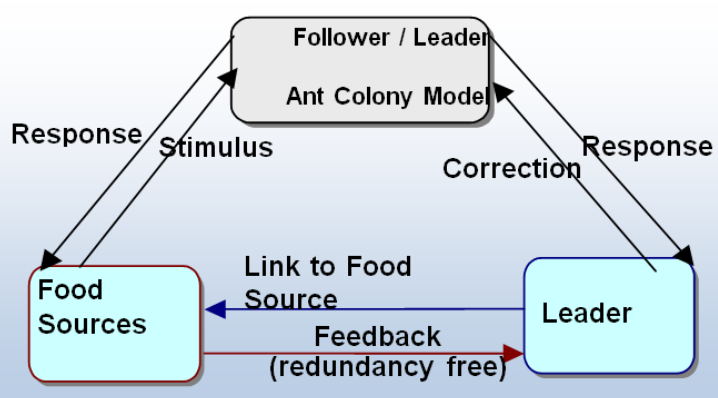

Fig. 12 A Schematic Diagram for Tandem Learning Bidirectional Feedback

\section{Tandem Running For A Pair Of Ants [32]}

Referring to the Figure 13, in below, it illustrates the interactive tandem learning process in accordance with the bidirectional communication relationship between the pair of two ants (leader and follower).

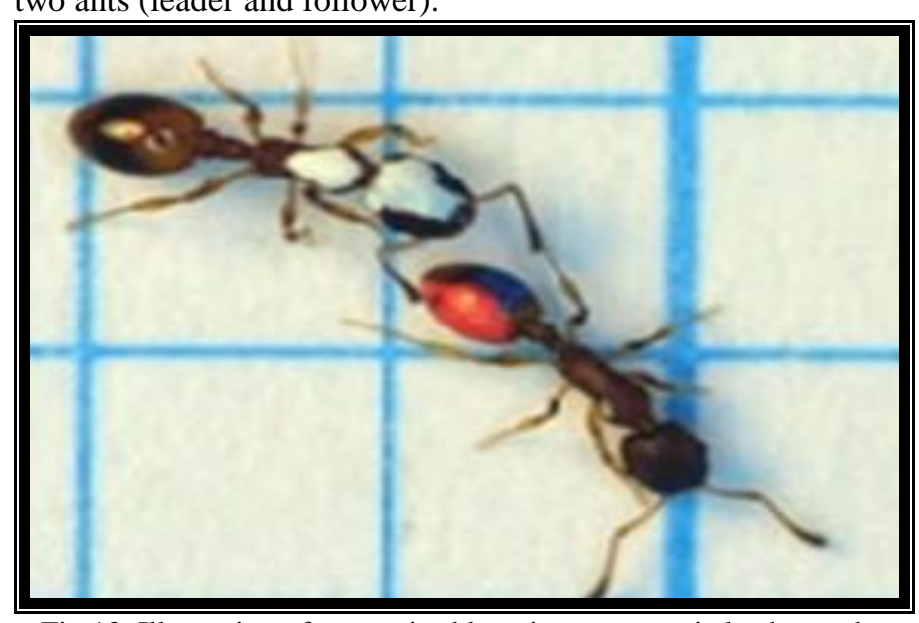

Fig.13. Illustration of supervised learning process via leader and follower Ants' interaction \{Adapted from [31] \}

Tandem running technique involves an interactive bidirectional feedback between teacher and pupil corresponding to leader and follower ants respectively. Furthermore, in this figure, depicted block named as (Follower/Leader Ant Colony Model) suggests that tandem follows after learning their lessons so well, that they can become tandem leaders. Tandem leaders have experience of the food source, whilst followers are naïve of its location. The leader proceeds towards the food source (red path) so long as the follower (blue path) maintains regular antennal contact with the leader's legs or abdomen. Progress of the tandem pair is slowed by frequent periods when the leader remains still whilst the follower performs a looped circuit, possibly to memorize landmarks along the path (points 1 and 3) [31][32]

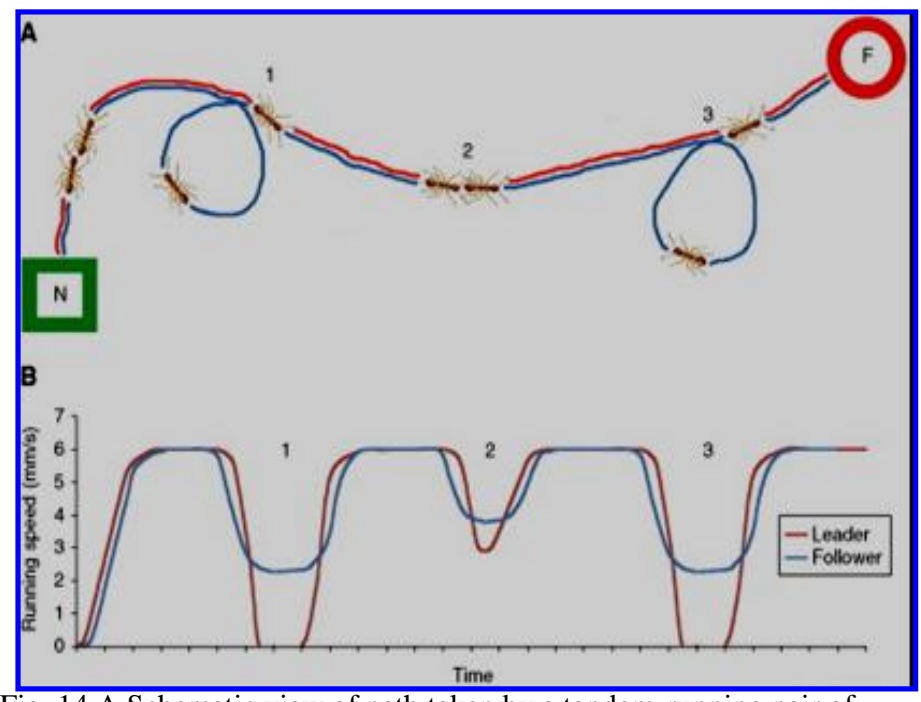

Fig. 14.A Schematic view of path taken by a tandem-running pair of Temnothorax albipennis ants from their nest $(\mathrm{N})$ to a food source $(\mathrm{F})$. (B) Running speed of leader (red line) and follower (blue line) during the same tandem-run.

Once this exploratory circuit is completed, and the follower re-establishes antennal contact, the leader continues onwards towards the food. If contact between follower and leader becomes less frequent during a tandem-run, the leader will slow down to allow the follower to catch up (point 2). Interestingly, Tandem leaders pay a cost because they would normally have reached the food around four times faster if not hampered by a follower. But the benefit is that the follower learns where the food is much quicker than it would have done independently. Tandem followers learn their lessons so well that they often become tandem leaders and in this way timesaving information flows through the ant colony. Referring to Figure 14. in below, it illustrates the path taken by tandem running pair of Temnothorax albipennis ants from their nest (Green Square) to food source (Red circle). The leader proceeds towards the food source (red path) so long as the follower (blue path) maintains regular antennal contact with the leader's legs or abdomen [31].

\section{Simulation Results}

\section{A. Simplified Simulation Program Flowchart}

Referring to Figure 15, a simplified macro-level flowchart for simulation program is introduced. It describes briefly the algorithmic steps for a suggested realistic simulation program of adopted Artificial Neural Networks' model taking into account the different number of neurons.(\# neurons). 


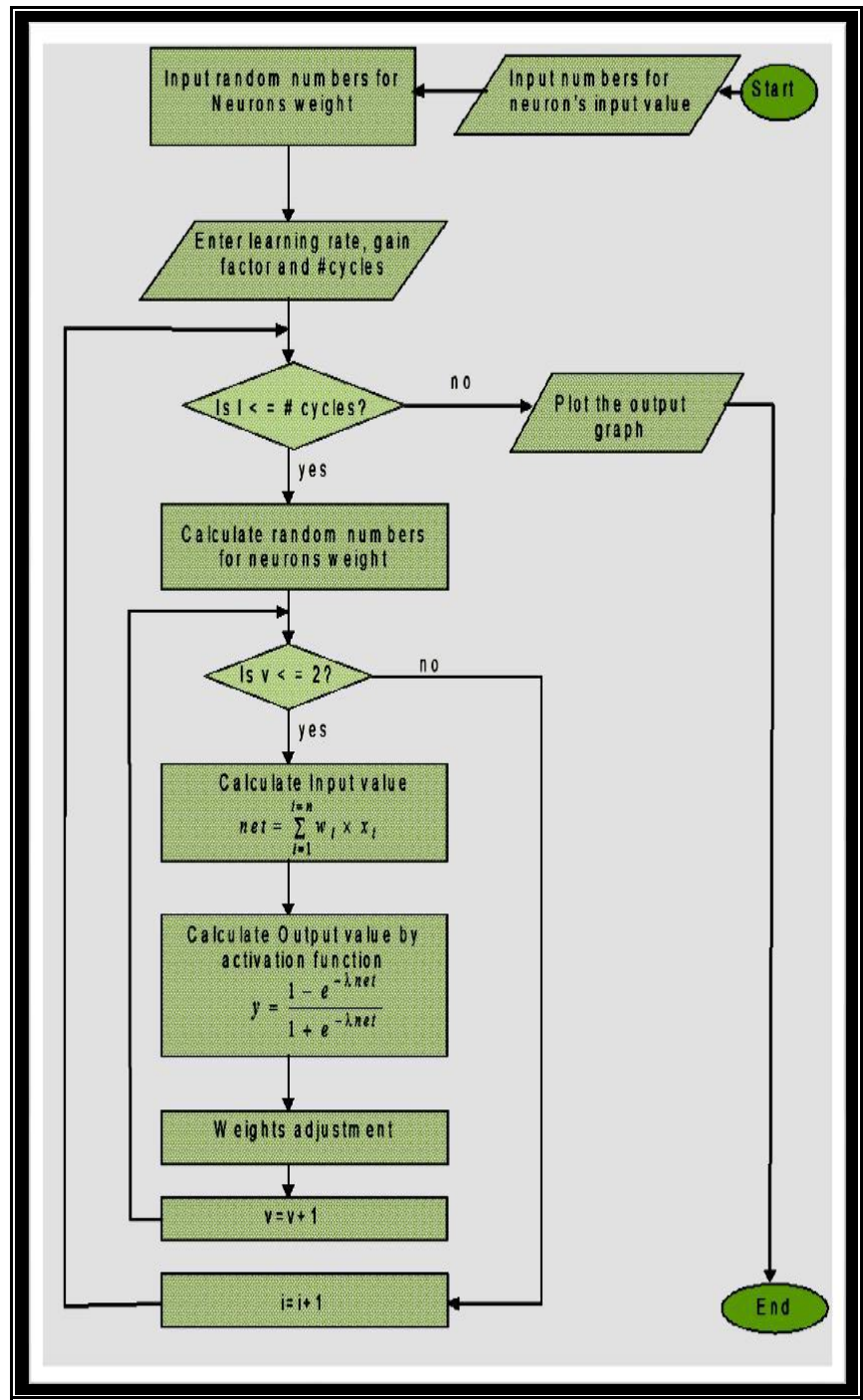

Fig. 15.A simplified macro level flowchart describing algorithmic steps (for various numbers of neurons) using Artificial Neural Networks modeling.

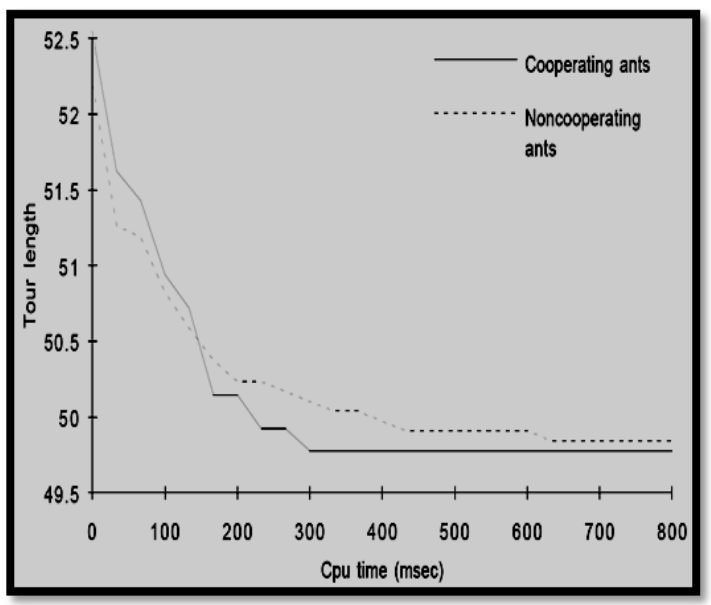

Fig.16. Cooperating ants find better solutions in a shorter time. Average on 25 runs. The number of ants was set to $m=4$, adapted from [25].

\section{B. Speed Versus Accuracy for Optimal Solution of TSP}

The convergence to solve optimally TSP is illustrated at Figure 16. Where it is shown in below, the relation between tour length versus the CPU time is given. It is observed the effect of ant cooperation level on reaching optimum (minimum tour). Obviously, as level of cooperation among ants increases (better communication among ants) the CPU time needed to reach optimum solution is decreased. So, that optimum solution is observed to be reached (with cooperation) after 300 (msec) CPU the while that solution is reached after $600(\mathrm{msec})$ CPU time (without cooperation).

In other words, by different levels of cooperation (communication among ants) the optimum solution is reached after CPU time $\tau$ placed somewhere between above two limits 300-650 (msec). Referring to [25],[27], cooperation among processing agents (ants) is a critical factor affecting ACS performance as illustrated at Figure 16. So, the number of ants required to get optimum solution differs in accordance with cooperation levels among ants. This number is analogous to number of trials in OCR process [24]. Moreover, the signal to noise ratio is observed to be directly proportional to leaning rate parameter in self-organized ANN models [18]. That implies the increase of stored experience due to learning by interaction with environment[23]. Additionally, by referring to Figure 17, the relation between various learning rate values and the relative error values is illustrated at[37]. More precisely, that figure presents the relation between relative error values $e_{k}(n)$ at any number of time cycles(n), versus the speed-accuracy trade-offs [38],considering the error correction learning while solving TSP.

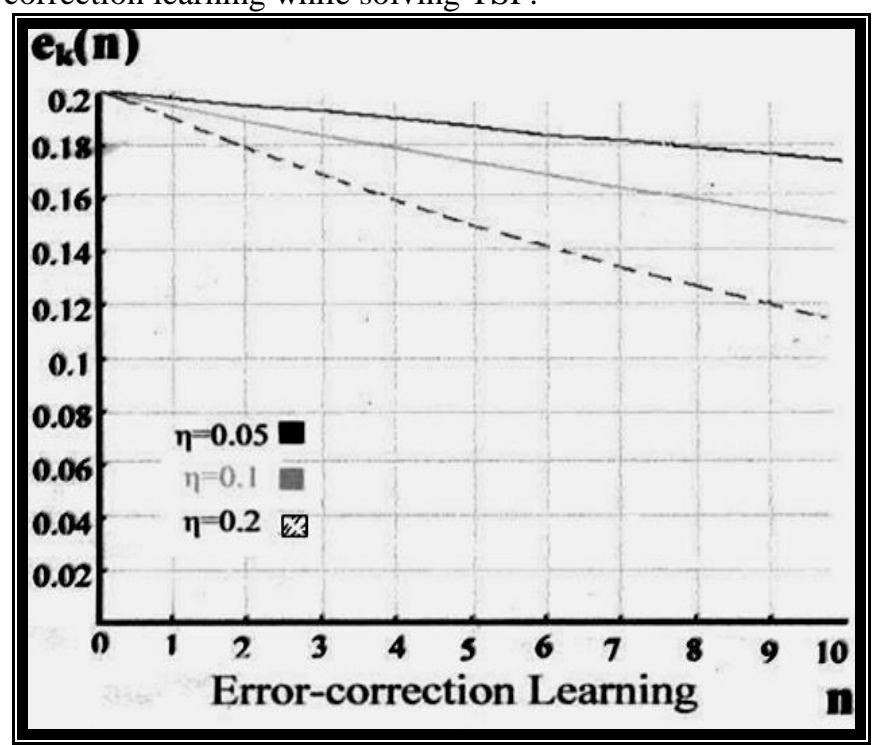

Fig. 17. Adaptability performance concerned with error correction learning algorithm \{adapted from [37] 
TABLE IV

ILLUSTRATES THE RELATION BETWEEN INCREASING \# NEURONS CONTRIBUTING FOR LEARNIN VESUS RESPONSE TIME AND AVARAGE LEARNING SPEED CONSIDERING ERROR CORRECTION LEARNING RULE.

\begin{tabular}{||l||c|c|c|c|c|c||}
\hline $\begin{array}{l}\text { Number of } \\
\text { Neurons } \\
\text { Contributing } \\
\text { Learning Process }\end{array}$ & 3 & 5 & 7 & 9 & 11 & 14 \\
\hline \hline $\begin{array}{l}\text { Measured Response } \\
\text { Time by (\# Cycles) }\end{array}$ & 79.7 & 61.8 & 36.9 & 19.8 & 14.8 & 7.3 \\
\hline \hline $\begin{array}{l}\text { Average Learning } \\
\text { Speed (1/Cycle). }\end{array}$ & 0.013 & 0.016 & 0.027 & 0.051 & 0.068 & 0.137 \\
\hline
\end{tabular}

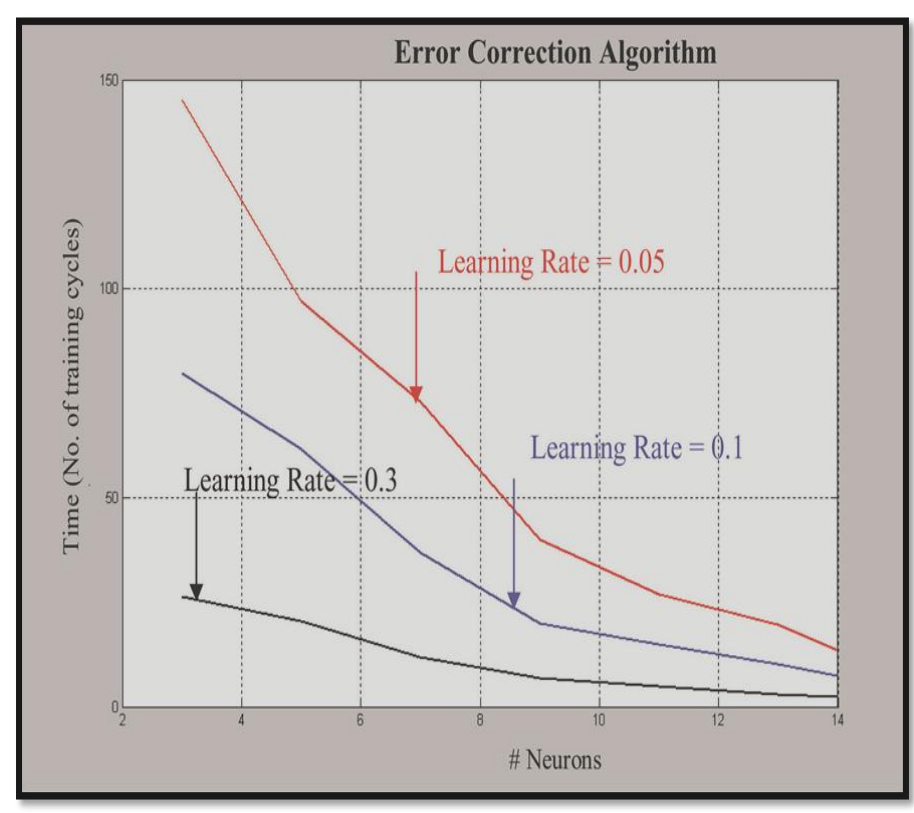

Fig. 18. Illustration of the error correction learning performance considering the tine response (Learning Convergence) for three learning rate value $(0.05,0.3$, and 0.5$)$

\section{CONCLUSIONS}

This piece of research comes to three interesting conclusive remarks presented as follows:

- The humble ant is not only capable of solving difficult mathematical problems, but is even able to do what few computer algorithms can - adapt the optimal solution to fit a changing problem, deepen our understanding of how even simple animals can overcome complex and dynamic problems in nature, and will help computer scientists develop even better software to solve logistical problems and maximize efficiency in many human industries.

- According to above presented two experimental works associated to the suggested problem of ACS problems based on tandem learning. And, the analysis and evaluation by ANNs modeling, observed to be in well correspondence and analogy with that optimization process performed on the basis of Swarm Intelligence (SI) by ACS. The optimal solution of TSP strategy in ants proved to reach its solution with minimum time rather than the other problem that reaches optimum average speed while solving TSP by referring to the analogous two figures $8 \& .9$. In addition to the analogy between two figures 11 , and 12 .

- Interestingly, the intercommunication levels among ants shown to be analogous to both learning parameters of ANN (Learning rates and the Gain factor values). Additionally, the number of ants (in ACS) is corresponding to the number of neurons(in ANN). Generally, the introduced approach is very beneficial for more realistic modeling of nonhuman (Social Insects) learning processes versus other realistic artificial neural networks' models, that for more applications in the future.

\section{REFERENCES}

[1] Nazmul Siddique, and Hojjat Adeli "Nature Inspired Computing: An Overview and Some Future Directions" Published online on November 30, 2015 doi: $10.1007 / \mathrm{s} 12559-015-9370-8$ PMCID: PMC4675795.Available online at: https://www.ncbi.nlm.nih.gov/pmc/articles/PMC4675795/

[2] Grzegorz Rozenberg, Thomas Back, and Joost N. Kok (Eds.) "A Hand Book of Natural Computing " Available online at: http://www.springer.com/978-3-540-92909-3

[3] Roger Highfield "Ants are in a class of their own with tandem teaching Ants are in a class of their own with tandem teaching". Available online on 12 Jan 2006: http://www.telegraph.co.uk/news/uknews/1507587/Ants-are-in-a-classof-their-own-withtandem-teaching.html

[4] Hassan M. H., et.al. "On Comparative Analogy between Ant Colony Systems and Neural Networks Considering Behavioural Learning Performance" Journal of Computer Sciences and Applications, 2015, Vol. 3, No. 3, 79-89 Available online at http://pubs.sciepub.com/jcsa/3/3/4 @ Science and Education Publishing DOI:10.12691/jcsa-3-3-4.

https://doi.org/10.12691/jcsa-3-3-4

[5] Hassan M. H. "Analytical Comparison of Swarm Intelligence Optimization versus Behavioral Learning Concepts Adopted by Neural Networks (An Overview) American Journal of Educational Research http://pubs.sciepub.com/education/3/7/2/index.html Vol. 3, No. 7, 2015, pp 800-806. doi: 0.12691/education-3-7-2.

[6] Hassan M. H. Mustafa, and Fadhel Ben Tourkia "On Comparative Analysis and Evaluation Of Social Insect Colonies' Behavior During Exploring Food Sources and Their Migration to A New Nest Versus Two of Neural Networks' Learning Paradigms. (Tandem Running Approach)" Published Journal IJATTMAS volume III issue xI nov 2017 Page 33-41

[7] Hassan M. H., et.al "Comparative Performance Analysis and Evaluation for One Selected Behavioral Learning System versus an Ant Colony Optimization System" Published at the Proceedings of the Second International Conference on Electrical, Electronics, Computer Engineering and their Applications (EECEA2015), Manila, Philippines, on Feb. 12-14, 2015.

[8] Maria Klatte, Kirstin Bergström, and Thomas Lachmann "Does noise affect learning? A short review on noise effects on cognitive performance in children". Available online 2013 Aug 30. doi: $10.3389 /$ fpsyg.2013.00578-at: https://www.ncbi.nlm.nih.gov/pmc/articles/PMC3757288/

[9] Ghoaimy M. A., et al. learning of Neural Networks using Noisy Data, Second International Conference On Artificial Intelligence Application, Cairo, Egypt, 389-399, Jan. 22-24, 1994.

[10] H.M.Hassan, "On Analysis and Evaluation of Non-Properly Prepared Teachers Based on Character Optical Recognition Considering Neural Networks Modeling" Published at Proceedings of the International Conference on Pattern Recognition and Image Processing ((ICPRIP'15) that held on March 16-17, 2015 Abu Dhabi (UAE).

[11] H.M.Hassan, and Ayoub Al-Hamadi "An Overview on Classrooms' Academic Performance Considering: Non-properly Prepared Instructors, Noisy Learning Environment, and Overcrowded Classes (Neural Networks' Approach)" has been accepted for oral presentation and publication at the upcoming 2015 6th International Conference on 
Distance Learning and Education (ICDLE 2015) in Pairs. Furthermore, this paper has been accepted to be published at the International Journal of Learning and Teaching in Vol.2, No.2, 2016 of IJLT.

[12] H.M.Hassan, et al "On Analysis And Evaluation of Multi-Sensory Cognitive Learning Of A Mathematical Topic Using Artificial Neural Networks", Journal of Telecommunications, 1(1), 99-104. (2010).

[13] H.M.Hassan "Quantifying of Learning Creativity Through Simulation and Modeling of Swarm Intelligence and Neural Networks", published at International Journal of Online Engineering iJOE-Volume 7, Issue 2, May 2011, pp.29-35.

[14] H.M.Hassan "On Quantifying Learning Creativity Using Artificial Neural Networks (A Mathematical Programming Approach) " published at CCCT 2007 conference held on July 12-17,2007Orlando,Florida,USA.

[15] H.M.Hassan "Analysis and Evaluation of Learning Creativity Phenomenon Using Artificial Neural Networks Modeling (A Quantitative Approach)", Published at the Sixth Annual Symposium on Learning and Technology, held on 26-27 April 28, 2008,Jedda Hilton Hotel.

[16] H.M.Hassan, et al "On Comparison Between Swarm Intelligence Optimization and Behavioral Learning Concepts Using Artificial Neural Networks (An over view)", published at the 12th World MultiConference on Systemics, Cybernetics and Informatics: WMSCI 2008 June 29th - July 2nd, 2008 - Orlando, Florida, USA.

[17] Kohonen T. "self-organization and Associative Memory": New York, Springer, 1984.

[18] Haykin S., Neural Networks, Englewood Cliffs, NJ: Prentice-Hall, 1999.

[19] J R Soc Interface. 2007 Apr 22; 4(13): 193-206. Published online 2006 Nov.28. doi: $10.1098 /$ rsif.2006.0177

[20] H.M. Hassan" On Simulation of Adaptive Learner Control Considering Students' Cognitive Styles Using Artificial Neural Networks (ANNs)" Published at CIMCA, Austria. 28-30 Nov.2005.

[21] D.O. Hebb, "The organization of behaviour", Wiley, New York (1949).

[22] Hassan. M. Mustafa and Ayoub Al-Hamadi "On Comparative Analogy of Academic Performance Quality Regarding Noisy Learning Environment versus Non-properly Prepared Teachers Using Neural Networks' Modeling" .Published in International Journal of Information and Education Technology, Vol. 6, No. 12, December 2016.

[23] M.Fukaya, et.al "Two level Neural Networks: Learning by Interaction with Environment", 1st ICNN, San Diego, (1988).

[24] Ghonaimy M.A., Al - Bassiouni, A.M. and Hassan, H.M "Leaning of Neural Networks Using Noisy Data". Second International Conference on Artificial Intelligence Applications, Cairo, Egypt, Jan 22-24, 1994.

[25] Alberto C., et al. Distributed optimization by ant colonies. Proceeding of ECAL91, Elsevier Publishing, pp 134-142, 1991.

[26] Yunlong Liu and Hiroki Yokota " Artificial ants deposit pheromone to search for regulatory DNA elements"

[27] .Available online at: https://bmcgenomics.biomedcentral.com/articles/10.1186/1471-2164-7221.Published: 30 August 2006.The-image-available-onlineat:http://media.springernature.com/full/springerstatic/image/art:10.1186/1471-2164-7221/MediaObjects/12864_2006_Article_604_Fig1_HTML.jpg

[28] E. Bonabeau, M. Dorigo, and G. Theraulaz. Swarm Intelligence: From Natural to Artificial Systems. Oxford University Press US,999.

[29] M. Dorigo and T. St $\square$ utzle. Ant Colony Optimization. MIT Press,2004.

[30] Hassan M. H. Mustafa, and Fadhel Ben Tourkia "On Comparative Analysis and Evaluation of Social Insect Colonies' Behavior During Exploring Food Sources and Their Migration to A New Nest Versus Two of Neural Networks' Learning Paradigms. (Tandem Running Approach)". Published at IJATTMAS: Nov, 2017, ISSN: 2454-5678 VOLUME-III, ISSUE-XI. Page 33-41.

[31] H. M. Hassan "On Evolutional Study of Comparative Analogy between Swarm Smarts and Neural Network Systems (Part 2)"Published at the 31 st International Conference for Statistics, Computer Science and Its Applications, Cairo-Egypt 1-6 April 2006.

[32] EllouiseLeadbeater Nigel E.RaineLarsChittka "Social Learning: Ants and the Meaning of Teaching". Available online: http://www.sciencedirect.com/science/article/pii/S0960982206014114.
[33] N.R. Franks, T. Richardson" Teaching in tandem-running ants" Nature, 439 (2006), p. 153. https://doi.org/10.1038/439153a

[34] Hassan M. H. Mustafa, and Fadhel Ben Tourkia On Analysis and Evaluation of Learning Creativity Quantification via Naturally Neural Networks' Simulation and Realistic Modeling of Swarm Intelligence" . Published at the proceeding of the conference: Eminent Association of Researchers in Engineering \& Technology(EARET), held in Kuala Lumpur, Malaysia, on 8-9 January 2018.

[35] Hassan M. H. Mustafa "On performance evaluation of brain based learning processes using neural networks," published at 2012 IEEE Symposium on Computers and Communications (ISCC), pp. 000672000679, 2012 IEEE Symposium on Computers and Communications (ISCC), 2012.

[36] Hassan M. H. Mustafa, and Fadhel Ben Tourkia "On Analysis And Evaluation Of Cocktail Party Effect On Applied Educational Practice Theory Using Neural Networks Modeling". Published at International Journal of Advanced Research (IJAR), on November 2017 : ISSN: 2320-5407 Int. J. Adv. Res. 5(11), 836-849

[37] H. M. Hassan. "Evaluation of Learning / Training Convergence Time Using Neural Network (ANNs)" published at, the Proceeding of 4th International Conference of Electrical Engineering ICEENG Conference, Military Technical College, Cairo, Egypt, pp.542-549, 2426 Nov. 2004.

[38] H.M. Mustafa, et al. "On Assessment of Brain Function Adaptability in Open Learning Systems Using Neural Network Modeling (Cognitive Styles Approach), Published at The IEEE International Conference on Communications and Information Technology ICCIT-2011, held on Mar 29, 2011 - Mar 31, 2011, Aqaba, Jordan. pp. 229-237.

[39] James A.R Marshall, Anna Dornhaus, Nigel R Franks, Tim Kovacs Noise, cost and speed-accuracy trade-offs: decision-making in a decentralized-system-published-22-April-2006.doi: 10.1098/rsif.2005.0075. Journal of Royal Society Interface Publishing http://rsif.royalsocietypublishing.org/content/3/7/243 